\title{
AN INTEGRAL OPERATOR PRESERVING $s$-CARLESON MEASURE ON THE UNIT BALL
}

\section{Cezhong Tong and Cheng Yuan*}

Hebei University of Technology, School of Science, Department of Mathematics

Tianjin, 300401, P. R. China; cezhongtong@hotmail.com

Tianjin University of Technology and Education, School of Science, Institute of Mathematics

Tianjin, 300222, P. R. China; yuancheng1984@163.com

\begin{abstract}
We establish an integral operator which preserves $s$-Carleson measure on the unit ball. As an application, we characterize the distance from Bloch-type functions to the analytic function space $F(p, q, s)$ on the ball.
\end{abstract}

\section{Introduction}

Let $\mathbf{B}_{n}$ be the unit ball of $\mathbf{C}^{n}$ with boundary $\mathbf{S}_{n}$ and $H\left(\mathbf{B}_{n}\right)$ the space of holomorphic functions on $\mathbf{B}_{n}$. When $n=1$, we have the unit disc $\mathbf{D}$.

If $\zeta \in \mathbf{S}_{n}$ and $r>0$, let $B(\zeta, r)=\left\{z \in \mathbf{B}_{n}:|1-\langle z, \zeta\rangle|<r\right\}$. For a constant $s>0$ and a positive Borel measure $\mu$ on $\mathbf{B}_{n}$, we call $\mu$ an $s$-Carleson measure if

$$
\|\mu\|_{\mathcal{C M}_{s}}=\sup \left\{\frac{\mu(B(\zeta, r))}{r^{n s}}: \zeta \in \mathbf{S}_{n}, r>0\right\}<\infty .
$$

We write $\mathcal{C M}_{s}$ for the class of all $s$-Carleson measures. When $s=1$, the $s$-Carleson measure becomes the classical Carleson measure on the ball. See [15] for more details. The Carleson measure plays a crucial role in lots of theories.

Motivated by Lemma 3.1.2 in [7] and Theorem 2.5 in [5], we investigate an integral operator which preserves $s$-Carleson measures on the unit ball. For $t, \lambda>0$, we define formally a linear operator $T_{t, \lambda}$ as

$$
T_{t, \lambda} f(z)=\int_{\mathbf{B}_{n}} \frac{\left(1-|w|^{2}\right)^{\lambda}}{|1-\langle z, w\rangle|^{t+\lambda}} f(w) \mathrm{d} v(w), \quad z \in \mathbf{B}_{n},
$$

where $\mathrm{d} v$ is the volume measure on $\mathbf{B}_{n}$ normalized with $v\left(\mathbf{B}_{n}\right)=1$ and $f \in H\left(\mathbf{B}_{n}\right)$. The main result of this manuscript shows that $\mathcal{C} \mathcal{M}_{s}$ is invariant under $T_{t, \lambda}$, which is stated as following:

Theorem 1. Assume $0<s \leq 1,1 \leq p<\infty$, and $\alpha>-1$. Let $\lambda>(\alpha+1-p) / p$, $t>n+1-(\alpha+1) / p$ and $f$ be Lebesgue measurable on $\mathbf{B}_{n}$. If $|f(z)|^{p}\left(1-|z|^{2}\right)^{\alpha} \mathrm{d} v(z)$ belongs to $\mathcal{C} \mathcal{M}_{s}$, then $\left|T_{t, \lambda} f(z)\right|^{p}\left(1-|z|^{2}\right)^{p(t-n-1)+\alpha} \mathrm{d} v(z)$ also belongs to $\mathcal{C M}_{s}$.

doi:10.5186/aasfm.2015.4017

2010 Mathematics Subject Classification: Primary 30H25, 30H30, 45P05.

Key words: Carleson measures, analytic $F(p, q, s)$ spaces, Bloch-type spaces.

*Corresponding author.

Cezhong Tong is supported by the National Natural Science Foundation of China (Grant Nos. 11301132 and 11171087) and Natural Science Foundation of Hebei Province (Grant No. A2013202265); Cheng Yuan is supported by NSFC 11226086 of China and Tianjin Advanced Education Development Fund 20111005. 
For $f \in H\left(\mathbf{B}_{n}\right)$ with homogeneous expansion

$$
f(z)=\sum_{k=0}^{\infty} f_{k}(z)
$$

the radial derivative of $f$ is defined as

$$
R f(z)=\sum_{k=1}^{\infty} k f_{k}(z) .
$$

It is easy to see that $R f \in H\left(\mathbf{B}_{n}\right)$ with

$$
f(z)-f(0)=\int_{0}^{1} \frac{R f(t z)}{t} \mathrm{~d} t
$$

For $0<\alpha<\infty$, the Bloch-type space on $\mathbf{B}_{n}$, denoted by $\mathcal{B}_{\alpha}$, is the space of analytic functions on $\mathbf{B}_{n}$ satisfying

$$
\|f\|_{\mathcal{B}_{\alpha}}=\sup _{z \in \mathbf{B}_{n}}\left(1-|z|^{2}\right)^{\alpha}|R f(z)|<\infty .
$$

It is well known that $\mathcal{B}_{\alpha}$ is a Banach space under the norm

$$
\|f\|_{\mathcal{B}_{\alpha}}^{*}=|f(0)|+\|f\|_{\mathcal{B}_{\alpha}} .
$$

In particular, $\mathcal{B}_{1}$ becomes the classic Bloch space $\mathcal{B}$, which is the maximal Möbius invariant Banach space.

For any point $a \in \mathbf{B}_{n} \backslash\{0\}$ we define

$$
\varphi_{a}(z)=\frac{a-P_{a}(z)-s_{a} Q_{a}(z)}{1-\langle z, a\rangle}, \quad z \in \mathbf{B}_{n}
$$

where $s_{a}=\sqrt{1-|a|^{2}}, P_{a}(z)=\langle z, a\rangle a /|a|^{2}$ and $Q_{a}(a)=z-P_{a}(z)$. When $a=0$, we simply define $\varphi_{a}(z)=-z$. It is easy to check that $\varphi_{a}(0)=a, \varphi_{a}(a)=0$, $\varphi_{a}\left(\varphi_{a}(z)\right)=z$ and $1-\left|\varphi_{a}(z)\right|^{2}=\left(1-|a|^{2}\right)\left(1-|z|^{2}\right) /|1-\langle z, a\rangle|^{2}$. All these basic facts can be found in [15].

Let $0<p<\infty, 0 \leq s<\infty,-1<q+s<\infty,-1<q+n<\infty$. The space $F(p, q, s)$, known as the general family of function spaces, is defined as the set of $f \in H\left(\mathbf{B}_{n}\right)$ for which

$$
\|f\|_{F(p, q, s)}^{p}=\sup _{a \in \mathbf{B}_{n}} \int_{\mathbf{B}_{n}}|R f(z)|^{p}\left(1-|z|^{2}\right)^{q}\left(1-\left|\varphi_{a}(z)\right|^{2}\right)^{s} \mathrm{~d} v(z)<\infty .
$$

The spaces $F(p, q, s)$ were first introduced by Zhao on $\mathbf{D}$ in [12]. Recently, Zhang, He and Cao characterized several equivalent norms of $F(p, q, s)$ on $\mathbf{B}_{n}$ in [11].

As the sequel of [10], this manuscript aims to characterize the distance from $f \in \mathcal{B}_{\alpha}$ to $F(p, q, s)$ on $\mathbf{B}_{n}$ as an application of Theorem 1. Let $X \subset \mathcal{B}_{\alpha}$ be an analytic function space. The distance from a Bloch-type function $f$ to $X$ is defined by

$$
\operatorname{dist}_{\mathcal{B}_{\alpha}}(f, X)=\inf _{g \in X}\|f-g\|_{\mathcal{B}_{\alpha}} .
$$

The second result of this paper is motivated by $[1,2,6,9,13]$, which states as following: 
Theorem 2. Suppose $1 \leq p<\infty, 0<s \leq n,-1<q+s<\infty$ and $f \in \mathcal{B}_{\frac{n+1+q}{p}}$. Then

$$
\operatorname{dist}_{\mathcal{B}_{\frac{n+1+q}{p}}}(f, F(p, q, s)) \approx \inf \left\{\varepsilon>0: \frac{\chi_{\widetilde{\Omega}_{\varepsilon}(f)}(z) \mathrm{d} v(z)}{\left(1-|z|^{2}\right)^{n+1-s}} \in \mathcal{C} \mathcal{M}_{\frac{s}{n}}\right\}
$$

where $\widetilde{\Omega}_{\varepsilon}(f)=\left\{z \in \mathbf{B}_{n}:\left(1-|z|^{2}\right)^{\frac{n+1+q}{p}}|R f(z)| \geq \varepsilon\right\}$ and $\chi_{\widetilde{\Omega}_{\varepsilon}(f)}$ is the characteristic function of the set $\widetilde{\Omega}_{\varepsilon}(f)$.

The argument in our proof of Theorem 2 is a generalization of [10], which follows from Theorem 3.1.3 in [7]. The distance from a $\mathcal{B}_{\alpha}$ function to Campanato-Morrey space on $\mathbf{D}$ was given in [8] with the similar idea.

Notation. Throughout this paper, we only write $U \lesssim V$ (or $V \gtrsim U$ ) for $U \leq c V$ for a positive constant $c$, and moreover $U \approx V$ for both $U \lesssim V$ and $V \lesssim U$.

\section{Preliminaries}

The following result is well-known, for example, see Theorem 50 in [14] for a proof.

Lemma 3. Let $s, \gamma \in(0, \infty)$ and $\mu$ be a nonnegative Borel measure on $\mathbf{B}_{n}$. Then $\mu \in \mathcal{C M}_{s}$ if and only if

$$
\|\mu\|_{\mathcal{C M}_{s}, \gamma}=\sup _{w \in \mathbf{B}_{n}} \int_{\mathbf{B}_{n}} \frac{\left(1-|w|^{2}\right)^{\gamma}}{|1-\langle z, w\rangle|^{\gamma+n s}} \mathrm{~d} \mu(z)<\infty .
$$

It is easy to check that if (2) holds for some $\gamma>0$, it holds for all $\gamma>0$. According to Lemma 3, the following corollary can be easily obtained.

Corollary 4. Let $f$ be an analytic function on $\mathbf{B}_{n}$. Then $f \in F(p, q, s)$ if and only if $|R f(z)|^{p}\left(1-|z|^{2}\right)^{q+s} \mathrm{~d} v(z)$ is an $s / n$-Carleson measure if and only if there exists an $\gamma>0$ such that

$$
\|f\|_{F(p, q, s), \gamma}^{p}=\sup _{w \in \mathbf{B}_{n}} \int_{\mathbf{B}_{n}} \frac{\left(1-|w|^{2}\right)^{\gamma}}{|1-\langle z, w\rangle|^{\gamma+s}}|R f(z)|^{p}\left(1-|z|^{2}\right)^{q+s} \mathrm{~d} v(z)<\infty .
$$

We also need the following standard result from [15].

Lemma 5. Suppose $t>-1$ and $c>0$. Then

$$
\int_{\mathbf{B}_{n}} \frac{\left(1-|w|^{2}\right)^{t}}{|1-\langle z, w\rangle|^{n+1+t+c}} \mathrm{~d} v(w) \approx \frac{1}{\left(1-|z|^{2}\right)^{c}}
$$

for all $z \in \mathbf{B}_{n}$.

The following lemma is quoted from [3], which is Lemma 2.5 there.

Lemma 6. Suppose $s>-1$ and $r, t>0$. If $t<s+n+1<r$, then

$$
\int_{\mathbf{B}_{n}} \frac{\left(1-|w|^{2}\right)^{s} \mathrm{~d} v(w)}{|1-\langle z, w\rangle|^{r}|1-\langle\eta, w\rangle|^{t}} \lesssim \frac{1}{\left(1-|z|^{2}\right)^{r-s-n-1}|1-\langle\eta, z\rangle|^{t}} .
$$

Next we show that $F(p, q, s)$ is contained in $\mathcal{B}_{\frac{n+1+q}{p}}$. Similar result on the disk can be found in [12].

Lemma 7. Suppose $1 \leq p<\infty, 0 \leq s<\infty$ and $\max \{-n-1,-s-1\}<q<\infty$, then $F(p, q, s) \subset \mathcal{B}_{\frac{n+1+q}{p}}$. Moreover, if $s>n$, then $F(p, q, s)=\mathcal{B}_{\frac{n+1+q}{p}}$. 
Proof. By using the reproducing formula on $R f$ we can get that

$$
R f(z)=\frac{\Gamma(n+1+\alpha)}{n ! \Gamma(\alpha+1)} \int_{\mathbf{B}_{n}} \frac{\left(1-|w|^{2}\right)^{\alpha} R f(w)}{(1-\langle z, w\rangle)^{n+1+\alpha}} \mathrm{d} v(w)
$$

for all $\alpha>-1$. In this proof we take $\alpha>\frac{q+s}{p}$ and $0<\gamma<n+1+q$.

When $p=1$, it is easy to check that

$$
\begin{aligned}
& \left(1-|z|^{2}\right)^{n+1+q}|R f(z)| \lesssim \int_{\mathbf{B}_{n}} \frac{\left(1-|z|^{2}\right)^{n+1+q}\left(1-|w|^{2}\right)^{\alpha}|R f(w)|}{|1-\langle z, w\rangle|^{n+1+\alpha}} \mathrm{d} v(w) \\
& =\int_{\mathbf{B}_{n}} \frac{\left(1-|w|^{2}\right)^{q+s}\left(1-|z|^{2}\right)^{\gamma}|R f(w)|}{|1-\langle z, w\rangle|^{\gamma+s}} \frac{\left(1-|z|^{2}\right)^{n+1+q-\gamma}\left(1-|w|^{2}\right)^{\alpha-q-s}}{|1-\langle z, w\rangle|^{n+1+\alpha-\gamma-s}} \mathrm{~d} v(w) \\
& \leq \int_{\mathbf{B}_{n}} \frac{\left(1-|z|^{2}\right)^{\gamma}}{|1-\langle z, w\rangle|^{\gamma+s}}|R f(w)|\left(1-|w|^{2}\right)^{q+s} \mathrm{~d} v(w) \\
& \quad \sup _{w \in \mathbf{B}_{n}} \frac{\left(1-|z|^{2}\right)^{n+1+q-\gamma}\left(1-|w|^{2}\right)^{\alpha-q-s}}{|1-\langle z, w\rangle|^{n+1+\alpha-\gamma-s}} \\
& \leq\|f\|_{F(p, q, s), \gamma} \sup _{w \in \mathbf{B}_{n}} \frac{\left(1-|z|^{2}\right)^{n+1+q-\gamma}\left(1-|w|^{2}\right)^{\alpha-q-s}}{|1-\langle z, w\rangle|^{n+1+\alpha-\gamma-s}} .
\end{aligned}
$$

Since $n+1+q-\gamma>0$ and $\alpha-q-s>0$, it follows that

$$
\sup _{w \in \mathbf{B}_{n}} \frac{\left(1-|z|^{2}\right)^{n+1+q-\gamma}\left(1-|w|^{2}\right)^{\alpha-q-s}}{|1-\langle z, w\rangle|^{n+1+\alpha-\gamma-s}} \lesssim 1
$$

Thus $F(p, q, s) \subset \mathcal{B}_{\frac{n+1+q}{p}}$ when $p=1$.

When $p>1$, take $p^{\prime}=p /(p-1)$. Then it follows from the Hölder's inequality that

$$
\begin{aligned}
|R f(z)| \lesssim & \int_{\mathbf{B}_{n}} \frac{\left(1-|w|^{2}\right)^{\frac{q+s}{p}}\left(1-|z|^{2}\right)^{\frac{\gamma}{p}}|R f(w)|}{|1-\langle z, w\rangle|^{\frac{s+\gamma}{p}}} \frac{\left(1-|z|^{2}\right)^{-\frac{\gamma}{p}}\left(1-|w|^{2}\right)^{\alpha-\frac{q+s}{p}}}{|1-\langle z, w\rangle|^{n+1+\alpha-\frac{s+\gamma}{p}}} \mathrm{~d} v(w) \\
\leq & \left(\int_{\mathbf{B}_{n}} \frac{\left(1-|z|^{2}\right)^{\gamma}}{|1-\langle z, w\rangle|^{s+\gamma}}|R f(w)|^{p}\left(1-|w|^{2}\right)^{q+s} \mathrm{~d} v(w)\right)^{\frac{1}{p}} \\
& \cdot \frac{1}{\left(1-|z|^{2}\right)^{\frac{\gamma}{p}}}\left(\int_{\mathbf{B}_{n}} \frac{\left(1-|w|^{2}\right)^{p^{\prime}\left(\alpha-\frac{q+s}{p}\right)}}{|1-\langle z, w\rangle|^{p^{\prime}\left(n+1+\alpha-\frac{s+\gamma}{p}\right)}} \mathrm{d} v(w)\right)^{\frac{1}{p^{\prime}}} \\
\leq & \frac{\|f\|_{F(p, q, s), \gamma}}{\left(1-|z|^{2}\right)^{\frac{\gamma}{p}}}\left(\int_{\mathbf{B}_{n}} \frac{\left(1-|w|^{2}\right)^{p^{\prime}\left(\alpha-\frac{q+s}{p}\right)}}{|1-\langle z, w\rangle|^{p^{\prime}\left(n+1+\alpha-\frac{s+\gamma}{p}\right)}} \mathrm{d} v(w)\right)^{\frac{1}{p^{\prime}}} \\
\lesssim & \|f\|_{F(p, q, s), \gamma} \frac{1}{\left(1-|z|^{2}\right)^{\frac{\gamma}{p}}}\left(\frac{1}{\left(1-|z|^{2}\right)^{\frac{n+1+q-\gamma}{p-1}}}\right) \\
= & \|f\|_{F(p, q, s), \gamma} \frac{1}{\left(1-|z|^{2}\right)^{\frac{n+1+q}{p}}}
\end{aligned}
$$

Apparently, Lemma 5 is applied in the last inequality. This gives that $F(p, q, s) \subset$ $\mathcal{B}_{\frac{n+1+q}{p}}$ when $1<p<\infty$. 
Now, suppose $s>n$, let $f \in \mathcal{B}_{\frac{n+1+q}{p}}$, then

$$
|R f(z)|\left(1-|z|^{2}\right)^{\frac{n+1+q}{p}} \leq\|f\|_{\mathcal{B}_{\frac{n+1+q}{p}}}<\infty
$$

for all $z \in \mathbf{B}_{n}$. It follows that

$$
\begin{aligned}
& \|f\|_{F(p, q, s)}^{p}=\sup _{a \in \mathbf{B}_{n}} \int_{\mathbf{B}_{n}}|R f(z)|^{p}\left(1-|z|^{2}\right)^{q+s}\left(\frac{1-|a|^{2}}{|1-\langle z, a\rangle|^{2}}\right)^{s} \mathrm{~d} v(z) \\
& =\sup _{a \in \mathbf{B}_{n}} \int_{\mathbf{B}_{n}}|R f(z)|^{p}\left(1-|z|^{2}\right)^{q+n+1}\left(1-|z|^{2}\right)^{s-n-1}\left(\frac{1-|a|^{2}}{|1-\langle z, a\rangle|^{2}}\right)^{s} \mathrm{~d} v(z) \\
& \leq\|f\|_{\mathcal{B}_{\frac{n+1+q}{p}}^{p}} \sup _{a \in \mathbf{B}_{n}}\left(1-|a|^{2}\right)^{s} \int_{\mathbf{B}_{n}} \frac{\left(1-|z|^{2}\right)^{s-n-1}}{|1-\langle z, a\rangle|^{2 s}} \mathrm{~d} v(z) \approx\|f\|_{\mathcal{B}_{\frac{n+1+q}{p}}^{p}}^{p}
\end{aligned}
$$

This completes the proof.

\section{Proof of Theorem 1}

Proof. When $p=1$, according to Lemma 3, it is sufficient to show that

$$
\sup _{a \in \mathbf{B}_{n}} \int_{\mathbf{B}_{n}} \frac{\left(1-|a|^{2}\right)^{\gamma}}{|1-\langle z, a\rangle|^{\gamma+n s}}\left|T_{t, \lambda} f(z)\right|\left(1-|z|^{2}\right)^{t-n-1+\alpha} \mathrm{d} v(z)<\infty
$$

for some $\gamma>0$. That is to show

$$
\sup _{a \in \mathbf{B}_{n}} \int_{\mathbf{B}_{n}} \frac{\left(1-|a|^{2}\right)^{\gamma}\left(1-|z|^{2}\right)^{t-n-1+\alpha}}{|1-\langle z, a\rangle|^{\gamma+n s}}\left|\int_{\mathbf{B}_{n}} \frac{\left(1-|w|^{2}\right)^{\lambda} f(w)}{|1-\langle z, w\rangle|^{t+\lambda}} \mathrm{d} v(w)\right| \mathrm{d} v(z)
$$

is finite. By Fubini's theorem, we need to verify that

$$
\sup _{a \in \mathbf{B}_{n}} \int_{\mathbf{B}_{n}} \frac{\left(1-|a|^{2}\right)^{\gamma}|f(w)|}{\left(1-|w|^{2}\right)^{-\lambda}} \int_{\mathbf{B}_{n}} \frac{\left(1-|z|^{2}\right)^{t-n-1+\alpha} \mathrm{d} v(z)}{|1-\langle z, w\rangle|^{t+\lambda}|1-\langle z, a\rangle|^{\gamma+n s}} \mathrm{~d} v(w)
$$

is finite.

Choose $\gamma$ such that $\gamma+n s<t+\alpha$. Notice that $t-n-1+\alpha>-1$ and $\lambda>\alpha$ in this case. Then by Lemma 6 the last integral can be controlled by

$$
\sup _{a \in \mathbf{B}_{n}} \int_{\mathbf{B}_{n}} \frac{\left(1-|a|^{2}\right)^{\gamma}}{|1-\langle w, a\rangle|^{\gamma+n s}}|f(w)|\left(1-|w|^{2}\right)^{\alpha} \mathrm{d} A(w) .
$$

The desired result follows from Lemma 3, since $|f(z)|\left(1-|z|^{2}\right)^{\alpha} \mathrm{d} v(z)$ is an $s$-Carleson measure.

When $1<p<\infty$, it is sufficient to show that

$$
\int_{B(\zeta, r)}\left|T_{t, \lambda} f(z)\right|^{p}\left(1-|z|^{2}\right)^{p(t-n-1)+\alpha} \mathrm{d} v(z) \lesssim r^{n s}
$$

holds for all $\zeta \in \mathbf{S}_{n}$ and $r>0$. 
For each fixed $r>0$, there exists a smallest $N_{r} \in \mathbf{N}$ such that $2^{N_{r}} r \geq 2$, which means that $B\left(\zeta, 2^{N_{r}} r\right)=\mathbf{B}_{n}$. So we can make the following estimates:

$$
\begin{aligned}
& \int_{B(\zeta, r)}\left|T_{t, \lambda} f(z)\right|^{p}\left(1-|z|^{2}\right)^{p(t-n-1)+\alpha} \mathrm{d} v(z) \\
& =\int_{B(\zeta, r)}\left|\int_{\mathbf{B}_{n}} \frac{\left(1-|w|^{2}\right)^{\lambda}}{|1-\langle z, w\rangle|^{t+\lambda}} f(w) \mathrm{d} v(w)\right|^{p}\left(1-|z|^{2}\right)^{p(t-n-1)+\alpha} \mathrm{d} v(z) \\
& =\int_{B(\zeta, r)}\left|\left(\int_{B(\zeta, 2 r)}+\int_{\mathbf{B}_{n} \backslash B(\zeta, 2 r)}\right) \frac{\left(1-|w|^{2}\right)^{\lambda}}{|1-\langle z, w\rangle|^{t+\lambda}} f(w) \mathrm{d} v(w)\right|^{p}\left(1-|z|^{2}\right)^{p(t-n-1)+\alpha} \mathrm{d} v(z) \\
& \lesssim \int_{B(\zeta, r)}\left(\int_{B(\zeta, 2 r)} \frac{\left(1-|w|^{2}\right)^{\lambda}|f(w)|}{|1-\langle z, w\rangle|^{t+\lambda}} \mathrm{d} v(w)\right)^{p}\left(1-|z|^{2}\right)^{p(t-n-1)+\alpha} \mathrm{d} v(z) \\
& +\int_{B(\zeta, r)}\left(\int_{\mathbf{B}_{n} \backslash B(\zeta, 2 r)} \frac{\left(1-|w|^{2}\right)^{\lambda}|f(w)|}{|1-\langle z, w\rangle|^{t+\lambda}} \mathrm{d} v(w)\right)^{p} \frac{\mathrm{d} v(z)}{\left(1-|z|^{2}\right)^{p(n+1-t)-\alpha}}=\operatorname{Int}_{1}+\operatorname{Int}_{2} .
\end{aligned}
$$

For $\mathrm{Int}_{1}$, consider the linear operator $T: L^{p}\left(\mathbf{B}_{n}, \mathrm{~d} v\right) \rightarrow L^{p}\left(\mathbf{B}_{n}, \mathrm{~d} v\right)$ defined by

$$
(T f)(z)=\int_{\mathbf{B}_{n}} K(z, w) f(w) \mathrm{d} v(w),
$$

where the kernel is given by

$$
K(z, w)=\frac{\left(1-|w|^{2}\right)^{\lambda-\alpha / p}\left(1-|z|^{2}\right)^{t-n-1+\alpha / p}}{|1-\langle z, w\rangle|^{t+\lambda}} .
$$

We can apply Schur's test (see e.g. [16]) to verify that $T$ is a bounded operator on $L^{p}\left(\mathbf{B}_{n}, \mathrm{~d} v\right)$. Indeed, if we take $p^{\prime}=p /(p-1)$ again and let $h(z)=\left(1-|z|^{2}\right)^{-\frac{1}{p p^{\prime}}}$, then it follows from Lemma 5 that

$$
\int_{\mathbf{B}_{n}} K(z, w) h^{p}(z) \mathrm{d} v(z) \lesssim h^{p}(w)
$$

and

$$
\int_{\mathbf{B}_{n}} K(z, w) h^{p^{\prime}}(w) \mathrm{d} v(w) \lesssim h^{p^{\prime}}(z)
$$

Accordingly, the integral operator $T$ is bounded from $L^{p}\left(\mathbf{B}_{n}, \mathrm{~d} v\right)$ to $L^{p}\left(\mathbf{B}_{n}, \mathrm{~d} v\right)$.

Now we rewrite Int $_{1}$ as

$$
\operatorname{Int}_{1}=\int_{B(\zeta, r)}\left(\int_{B(\zeta, 2 r)} K(z, w)|f(w)|\left(1-|w|^{2}\right)^{\alpha / p} \mathrm{~d} v(w)\right)^{p} \mathrm{~d} v(z),
$$

and let

$$
g(w)=|f(w)|\left(1-|w|^{2}\right)^{\alpha / p} \chi_{B(\zeta, 2 r)}(w),
$$

where $\chi_{E}$ stands for the characteristic function of $E$. Recall that $|f(w)|^{p}\left(1-|w|^{2}\right)^{\alpha} \mathrm{d} v(w)$ is an $s$-Carleson measure, we have

$$
\|g\|_{L^{p}}^{p}=\int_{B(\zeta, 2 r)}|f(w)|^{p}\left(1-|w|^{2}\right)^{\alpha} \mathrm{d} v(w) \lesssim(2 r)^{n s} \lesssim r^{n s}
$$


Thus, we get

$$
\operatorname{Int}_{1} \lesssim \int_{\mathbf{B}_{n}}\left|\int_{\mathbf{B}_{n}} K(z, w) g(w) \mathrm{d} v(w)\right|^{p} \mathrm{~d} v(z)=\|T g\|_{L^{p}}^{p} \lesssim\|g\|_{L^{p}}^{p} \lesssim r^{n s}
$$

as desired.

To handle $\mathrm{Int}_{2}$, we note first that for $k=2,3, \cdots, N_{r}$, the inequality $|1-\langle z, w\rangle| \gtrsim$ $2^{k} r$ holds for $z \in B(\zeta, r)$ and $w \in B\left(\zeta, 2^{k} r\right) \backslash B\left(\zeta, 2^{k-1} r\right)$. For fixed $c>-1$, if we write $Q(\zeta, r)=\left\{\xi \in \mathbf{S}_{n}:|1-\langle\zeta, \xi\rangle|<r\right\}$ and denote $\sigma$ the normalized surface measure on $\mathbf{S}_{n}$, then a straightforward computation shows that

$$
\int_{B\left(\zeta, 2^{k} r\right)}\left(1-|z|^{2}\right)^{c} \mathrm{~d} v(z) \lesssim \int_{Q\left(\zeta, 2^{k} r\right)} \mathrm{d} \sigma \int_{1-2^{k} r}^{1} 2 n t^{2 n-1}\left(1-t^{2}\right)^{c} \mathrm{~d} t \lesssim\left(2^{k} r\right)^{n+1+c} .
$$

Notice that

$$
\mathbf{B}_{n} \backslash B(\zeta, 2 r)=\bigcup_{k=1}^{N_{r}-1} B\left(\zeta, 2^{k+1} r\right) \backslash B\left(\zeta, 2^{k} r\right)
$$

Since $p(t-n-1)+\alpha>-1$, we have

$$
\begin{aligned}
\operatorname{Int}_{2} & \lesssim \int_{B(\zeta, r)}\left(\sum_{k=1}^{N_{r}-1} \int_{B\left(\zeta, 2^{k+1} r\right) \backslash B\left(\zeta, 2^{k} r\right)} \frac{\left(1-|w|^{2}\right)^{\lambda}|f(w)|}{|1-\langle z, w\rangle|^{t+\lambda}} \mathrm{d} v(w)\right)^{p} \frac{\mathrm{d} v(z)}{\left(1-|z|^{2}\right)^{p(n+1-t)-\alpha}} \\
& \lesssim \int_{B(\zeta, r)}\left(\sum_{k=1}^{N_{r}-1} \int_{B\left(\zeta, 2^{k+1} r\right) \backslash B\left(\zeta, 2^{k} r\right)} \frac{\left(1-|w|^{2}\right)^{\lambda}|f(w)|}{\left(2^{k} r\right)^{t+\lambda}} \mathrm{d} v(w)\right)^{p} \frac{\mathrm{d} v(z)}{\left(1-|z|^{2}\right)^{p(n+1-t)-\alpha}} \\
& \lesssim r^{n+1+p(t-n-1)+\alpha}\left(\sum_{k=1}^{N_{r}-1} \int_{B\left(\zeta, 2^{k+1} r\right)} \frac{\left(1-|w|^{2}\right)^{\lambda}|f(w)|}{\left(2^{k} r\right)^{t+\lambda}} \mathrm{d} v(w)\right)^{p} .
\end{aligned}
$$

Keep in mind that $|f(w)|^{p}\left(1-|w|^{2}\right)^{\alpha} \mathrm{d} v(w)$ is an $s$-Carleson measure and $\lambda>(1+$ $\alpha-p) / p$, we can use the Hölder's inequality to get that

$$
\begin{aligned}
& \int_{B\left(\zeta, 2^{k+1} r\right)}\left(1-|w|^{2}\right)^{\lambda}|f(w)| \mathrm{d} v(w) \\
& \leq\left(\int_{B\left(\zeta, 2^{k+1} r\right)}\left(1-|w|^{2}\right)^{\alpha}|f(w)|^{p} \mathrm{~d} v(w)\right)^{\frac{1}{p}}\left(\int_{B\left(\zeta, 2^{k+1} r\right)}\left(1-|w|^{2}\right)^{\left(\lambda-\frac{\alpha}{p}\right) p^{\prime}} \mathrm{d} v(w)\right)^{\frac{1}{p^{\prime}}} \\
& \lesssim\left(2^{k+1} r\right)^{\frac{n s}{p}} \times\left(2^{k+1} r\right)^{\left(\lambda p^{\prime}-\frac{p^{\prime}}{p} \alpha+n+1\right) \frac{1}{p^{\prime}}} .
\end{aligned}
$$

Therefore, we can conclude that

$$
\begin{aligned}
\operatorname{Int}_{2} & \lesssim r^{n+1+p(t-n-1)+\alpha}\left(\sum_{k=1}^{\infty} \frac{\left(2^{k+1} r\right)^{\frac{n s}{p}} \times\left(2^{k+1} r\right)^{\left(\lambda p^{\prime}-\frac{p^{\prime}}{p} \alpha+n+1\right) \frac{1}{p^{\prime}}}}{\left(2^{k} r\right)^{t+\lambda}}\right)^{p} \\
& \lesssim r^{n s}\left(\sum_{k=1}^{\infty} 2^{k\left(\frac{n s-\alpha+(p-1)(n+1)}{p}-t\right)}\right)^{p} .
\end{aligned}
$$

The assumptions $t>n+1-\frac{\alpha+1}{p}$ and $0<s \leq 1$ imply that $t>\frac{n s-\alpha+(p-1)(n+1)}{p}$. This completes the proof. 


\section{Proof of Theorem 2}

Proof. Firstly, we prove

$$
\operatorname{dist}_{\mathcal{B}_{\frac{n+1+q}{p}}}(f, F(p, q, s)) \lesssim \inf \left\{\varepsilon>0: \frac{\chi_{\widetilde{\Omega}_{\varepsilon}(f)}(z) \mathrm{d} v(z)}{\left(1-|z|^{2}\right)^{n+1-s}} \in \mathcal{C} \mathcal{M}_{\frac{s}{n}}\right\} .
$$

When $\alpha>-1$, for $f \in \mathcal{B}_{\frac{n+1+q}{p}}, R f(z)$ can be rewritten as

$$
\int_{\mathbf{B}_{n}} \frac{R f(w) \mathrm{d} v_{\alpha}(w)}{(1-\langle z, w\rangle)^{n+1+\alpha}}
$$

where

$$
\mathrm{d} v_{\alpha}(z)=\frac{\Gamma(n+1+\alpha)}{n ! \Gamma(\alpha+1)}\left(1-|z|^{2}\right)^{\alpha} \mathrm{d} v(z) .
$$

Similarly as [15] and [9], it follows from $R f(0)=0$ that

$$
R f(z)=\int_{\mathbf{B}_{n}} R f(w)\left(\frac{1}{(1-\langle z, w\rangle)^{n+1+\alpha}}-1\right) \mathrm{d} v_{\alpha}(w)
$$

for all $z \in \mathbf{B}_{n}$. According to (1),

$$
f(z)-f(0)=\int_{0}^{1} \frac{R f(t z)}{t} \mathrm{~d} t=\int_{\mathbf{B}_{n}} R f(w) L(w, z) \mathrm{d} v_{\alpha}(w),
$$

where the kernel

$$
L(z, w)=\int_{0}^{1}\left(\frac{1}{(1-t\langle z, w\rangle)^{n+1+\alpha}}-1\right) \frac{\mathrm{d} t}{t} .
$$

Define

$$
f_{1}(z)=f(0)+\int_{\widetilde{\Omega}_{\varepsilon}(f)} R f(w) L(z, w) \mathrm{d} v_{\alpha}(w)
$$

and

$$
f_{2}(z)=\int_{\mathbf{B}_{n} \backslash \widetilde{\Omega}_{\varepsilon}(f)} R f(w) L(z, w) \mathrm{d} v_{\alpha}(w)
$$

Then

$$
f(z)=f_{1}(z)+f_{2}(z) .
$$

We can just verify that $\frac{\chi_{\tilde{\Omega}_{\epsilon}(f)}(z) \mathrm{d} v(z)}{\left(1-|z|^{2}\right)^{n+1-s}} \in \mathcal{C} \mathcal{M}_{\frac{s}{n}}$ implies $f_{1} \in F(p, q, s)$ and $f_{2} \in \mathcal{B}_{\frac{n+1+q}{p}}$ with $\left\|f_{2}\right\|_{\mathcal{B}_{\frac{n+1+q}{p}}} \lesssim \varepsilon$.

When $w$ is fixed, $L(z, w)$ becomes a holomorphic function in $z$. And it is easy to check that

$$
R L(z, w)=\frac{1}{(1-\langle z, w\rangle)^{n+1+\alpha}}-1
$$

and

$$
|R L(z, w)| \lesssim \frac{1}{|1-\langle z, w\rangle|^{n+1+\alpha}}
$$


We choose $\alpha=\frac{n+1+q}{p}$, then

$$
\begin{aligned}
\left|R f_{1}(z)\right| & =\left|\int_{\widetilde{\Omega}_{\varepsilon}(f)} R f(w) R L(z, w) \mathrm{d} v_{\alpha}(w)\right| \\
& \lesssim \int_{\widetilde{\Omega}_{\varepsilon}(f)}|R f(w)|\left(1-|w|^{2}\right)^{\frac{n+1+q}{p}}|R L(z, w)| \mathrm{d} v(w) \\
& \lesssim\|f\|_{\mathcal{B}_{\frac{n+1+q}{p}}} \int_{\mathbf{B}_{n}} \chi_{\widetilde{\Omega}_{\varepsilon}(f)}(w)|R L(z, w)| \mathrm{d} v(w) \\
& \lesssim\|f\|_{\mathcal{B}_{\frac{n+1+q}{p}}} \int_{\mathbf{B}_{n}} \frac{\chi_{\widetilde{\Omega}_{\varepsilon}(f)}(w)}{|1-\langle z, w\rangle|^{n+1+\frac{n+1+q}{p}}} \mathrm{~d} v(w) \\
& =\|f\|_{\mathcal{B}_{\frac{n+1+q}{p}}} \int_{\mathbf{B}_{n}} \frac{\left(1-|w|^{2}\right)^{\frac{n+1}{p}}}{|1-\langle z, w\rangle|^{n+1+\frac{n+1+q}{p}}} \frac{\chi_{\widetilde{\Omega}_{\varepsilon}(f)}(w)}{\left(1-|w|^{2}\right)^{\frac{n+1}{p}}} \mathrm{~d} v(w) .
\end{aligned}
$$

If we write

then

$$
g(w)=\frac{\chi_{\widetilde{\Omega}_{\varepsilon}(f)}(w)}{\left(1-|w|^{2}\right)^{\frac{n+1}{p}}}
$$

So, if

$$
|g(w)|^{p}\left(1-|w|^{2}\right)^{s} \mathrm{~d} v(w)=\chi_{\widetilde{\Omega}_{\varepsilon}(f)}(w)\left(1-|w|^{2}\right)^{s-n-1} \mathrm{~d} v(w) .
$$

$$
\chi_{\widetilde{\Omega}_{\varepsilon}(f)}(z)\left(1-|z|^{2}\right)^{s-n-1} \mathrm{~d} v(z)
$$

is in $\mathcal{C} \mathcal{M}_{\frac{s}{n}}$, Theorem 1 with $\lambda=\frac{n+1}{p}$ and $t=n+1+\frac{q}{p}$ implies that

$$
\left|R f_{1}(z)\right|^{p}\left(1-|z|^{2}\right)^{q+s} \mathrm{~d} v(z)
$$

belongs to $\mathcal{C} \mathcal{M}_{\frac{s}{n}}$. This means $f_{1} \in F(p, q, s)$. Meanwhile, we have

$$
\left|R f_{2}(z)\right| \lesssim \varepsilon \int_{\mathbf{B}_{n}} \frac{\mathrm{d} v(w)}{|1-\langle z, w\rangle|^{n+1+\frac{n+1+q}{p}}} \approx \frac{\varepsilon}{\left(1-|z|^{2}\right)^{\frac{n+1+q}{p}}} .
$$

This gives that $f_{2} \in \mathcal{B}_{\frac{n+1+q}{p}}$ with $\left\|f_{2}\right\|_{\mathcal{B}_{\frac{n+1+q}{p}}} \lesssim \varepsilon$. Thus we verified (4).

In order to prove the converse inequality of (4), we assume that

$$
\operatorname{dist}_{\mathcal{B}_{\frac{n+1+q}{p}}}(f, F(p, q, s))<\inf \left\{\varepsilon>0: \frac{\chi_{\widetilde{\Omega}_{\varepsilon}(f)}(z) \mathrm{d} v(z)}{\left(1-|z|^{2}\right)^{n+1-s}} \in \mathcal{C} \mathcal{M}_{\frac{s}{n}}\right\} .
$$

For short, let $\varepsilon_{0}$ denote the right-hand quantity of the last inequality. We only consider the case $\varepsilon_{0}>0$. Then there exists an $\varepsilon_{1}$ such that

$$
0<\varepsilon_{1}<\varepsilon_{0} \quad \text { and } \quad \operatorname{dist}_{\mathcal{B}_{\frac{n+1+q}{p}}}(f, F(p, q, s))<\varepsilon_{1} .
$$

Hence, we can find a $h \in F(p, q, s)$ such that

$$
\|f-h\|_{\frac{\mathcal{B}_{n+1+q}}{p}}<\varepsilon_{1} .
$$

Now for any $\varepsilon \in\left(\varepsilon_{1}, \varepsilon_{0}\right)$ we have that

$$
\chi_{\widetilde{\Omega}_{\varepsilon}(f)}(z)\left(1-|z|^{2}\right)^{s-n-1} \mathrm{~d} v(z)
$$

is not in $\mathcal{C M}_{\frac{s}{n}}$. But, $\|f-h\|_{\mathcal{B}_{\frac{n+1+q}{p}}}<\varepsilon_{1}$ yields

$$
\left(1-|z|^{2}\right)^{\frac{n+1+q}{p}}|R h(z)|>\left(1-|z|^{2}\right)^{\frac{n+1+q}{p}}|R f(z)|-\varepsilon_{1}, \quad \forall z \in \mathbf{B}_{n},
$$


and so

$$
\chi_{\widetilde{\Omega}_{\varepsilon}(f)}(z) \leq \chi_{\widetilde{\Omega}_{\varepsilon-\varepsilon_{1}}(h)}(z) \quad \forall z \in \mathbf{B}_{n}
$$

This implies that

$$
\chi_{\widetilde{\Omega}_{\varepsilon-\varepsilon_{1}}(h)}(z)\left(1-|z|^{2}\right)^{s-n-1} \mathrm{~d} v(z)
$$

does not belong to $\mathcal{C} \mathcal{M}_{\frac{s}{n}}$. On the other hand,

$$
\begin{aligned}
\chi_{\widetilde{\Omega}_{\varepsilon-\varepsilon_{1}}(h)}(z)\left(1-|z|^{2}\right)^{s-n-1} \mathrm{~d} v(z) & =\chi_{\widetilde{\Omega}_{\varepsilon-\varepsilon_{1}}(h)}(z) \frac{\left(1-|z|^{2}\right)^{q+s}}{\left(1-|z|^{2}\right)^{q+n+1}} \mathrm{~d} v(z) \\
& \leq \frac{|R h(z)|^{p}}{\left(\varepsilon-\varepsilon_{1}\right)^{p}}\left(1-|z|^{2}\right)^{q+s} \chi_{\widetilde{\Omega}_{\varepsilon-\varepsilon_{1}}(h)}(z) \mathrm{d} v(z) \\
& \leq \frac{1}{\left(\varepsilon-\varepsilon_{1}\right)^{p}}|\operatorname{Rh}(z)|^{p}\left(1-|z|^{2}\right)^{q+s} \mathrm{~d} v(z) .
\end{aligned}
$$

Since $h \in F(p, q, s)$,

$$
|R h(z)|^{p}\left(1-|z|^{2}\right)^{q+s} \mathrm{~d} v(z)
$$

is in $\mathcal{C M}_{\frac{s}{n}}$, and consequently

$$
\chi_{\widetilde{\Omega}_{\varepsilon-\varepsilon_{1}}(h)}(z)\left(1-|z|^{2}\right)^{s-n-1} \mathrm{~d} v(z)
$$

is in $\mathcal{C M}_{\frac{s}{n}}$. Now, a contradiction occurs. Thus we must have

$$
\varepsilon_{0} \leq \operatorname{dist}_{\frac{\mathcal{B}_{\frac{n+1+q}{p}}}{p}}(f, F(p, q, s)) \lesssim \varepsilon_{0}
$$

as required.

\section{Further remarks}

For a measurable function $f$ on $\mathbf{B}_{n}$, define the projection operator

$$
P_{t, \lambda} f(z)=\int_{\mathbf{B}_{n}} \frac{\left(1-|w|^{2}\right)^{\lambda}}{(1-\langle z, w\rangle)^{t+\lambda}} f(w) \mathrm{d} v(w), \quad z \in \mathbf{B}_{n} .
$$

In particular, if $\lambda>0$ and $t=n+1, P_{t, \lambda}$ is called the Bergman projection. It is shown in [15] that the Bergman projection is bounded from $L^{p}\left(\mathbf{B}_{n}, \mathrm{~d} v_{\lambda}\right)$ onto the Bergman space $A_{\lambda}^{p}$ when $1<p<\infty$.

When $1 \leq p<\infty, \alpha>-1,0<s \leq 1$, we define a class $\mathcal{G}_{p, \alpha, s}$ of measurable functions on $\mathbf{B}_{n}$ such that

$$
|f(z)|^{p}\left(1-|z|^{2}\right)^{\alpha} \mathrm{d} v(z) \in \mathcal{C} \mathcal{M}_{s} .
$$

Then, $f \in F(p, q, s)$ if and only if $R f \in \mathcal{G}_{p, q+s, \frac{s}{n}} \cap H\left(\mathbf{B}_{n}\right)$. The next theorem shows that the Bergman projection is bounded from $\mathcal{G}_{p, \alpha, s}$ to $\mathcal{G}_{p, \alpha, s} \cap H\left(\mathbf{B}_{n}\right)$.

Theorem 8. Let $1 \leq p<\infty, \alpha>-1,0<s \leq 1$. The Bergman projection $P_{n+1, \lambda}$ is a bounded linear operator from $\mathcal{G}_{p, \alpha, s}$ to $\mathcal{G}_{p, \alpha, s} \cap H\left(\mathbf{B}_{n}\right)$.

Proof. It can be easily checked that for all measurable $f$,

$$
\left|P_{t, \lambda} f\right| \leq\left|T_{t, \lambda} f\right| .
$$

Then Theorem 1 implies the desired result.

Further, we have the following corollary. 
Corollary 9. Let $1 \leq p<\infty, \alpha>-1$ and $0<s \leq 1$. Suppose $\lambda>(\alpha+1-p) / p$ and $t>n+1-(\alpha+1) / p$. Then the projection $P_{t, \lambda}$ is a bounded linear operator from $\mathcal{G}_{p, \alpha, s}$ to $\mathcal{G}_{p, p(t-n-1)+\alpha, s} \cap H\left(\mathbf{B}_{n}\right)$.

For an $s$-Carleson measure $\mu$ on $\mathbf{B}_{n}$, if

$$
\lim _{r \rightarrow 1} \frac{\mu(B(\zeta, r))}{r^{n s}}=0
$$

for $\zeta \in \mathbf{S}_{n}$ uniformly, we call $\mu$ a vanishing $s$-Carleson measure.

The following result is well-known. See, for example, the remark after Theorem 50 in [14].

Corollary 10. Let $s, \gamma \in(0, \infty)$ and $\mu$ be nonnegative Borel measures on $\mathbf{B}_{n}$. Then $\mu$ is a vanishing $s$-Carleson measure if and only if

$$
\lim _{|w| \rightarrow 1} \int_{\mathbf{B}_{n}} \frac{\left(1-|w|^{2}\right)^{\gamma}}{|1-\langle z, w\rangle|^{\gamma+n s}} \mathrm{~d} \mu(z)=0 .
$$

By a slight modification of the proof of Theorem 1, we can obtain the following result.

Lemma 11. Assume $0<s \leq 1,1 \leq p<\infty$, and $\alpha>-1$. Let $\lambda>(\alpha+1-p) / p$, $t>n+1-(\alpha+1) / p$ and $f$ be Lebesgue measurable on $\mathbf{B}_{n}$. If $|f(z)|^{p}\left(1-|z|^{2}\right)^{\alpha} \mathrm{d} v(z)$ is a vanishing $s$-Carleson measure, then

$$
\left|T_{t, \lambda} f(z)\right|^{p}\left(1-|z|^{2}\right)^{p(t-n-1)+\alpha} \mathrm{d} v(z)
$$

is also a vanishing $s$-Carleson measure.

For $0<\alpha<\infty$, the little Bloch-type space on $\mathbf{B}_{n}$, denoted by $\mathcal{B}_{\alpha}^{0}$, is the subspace of $\mathcal{B}_{\alpha}$ consisting of all $f \in \mathcal{B}_{\alpha}$ such that

$$
\lim _{|z| \rightarrow 1}\left(1-|z|^{2}\right)^{\alpha}|R f(z)|=0,
$$

and the space $F_{0}(p, q, s)$, is the subspace of $F(p, q, s)$ consisting of all $f \in F(p, q, s)$ such that

$$
\sup _{|a| \rightarrow 1} \int_{\mathbf{B}_{n}}|R f(z)|^{p}\left(1-|z|^{2}\right)^{q}\left(1-\left|\varphi_{a}(z)\right|^{2}\right)^{s} \mathrm{~d} v(z)=0 .
$$

Similar to Lemma 7, we have the following corollary.

Lemma 12. Suppose $1 \leq p<\infty, 0 \leq s<\infty$ and $\max \{-n-1,-s-1\}<$ $q<\infty$. If $f \in H\left(\mathbf{B}_{n}\right)$, then $f \in F_{0}(p, q, s)$ if and only if $|R f(z)|^{p}\left(1-|z|^{2}\right)^{q+s} \mathrm{~d} v(z)$ is a vanishing $\frac{s}{n}$-Carleson measure. Further, $F_{0}(p, q, s) \subset \mathcal{B}_{\frac{n+1+q}{p}}^{0}$. When $s>n$, $F_{0}(p, q, s)=\mathcal{B}_{\frac{n+1+q}{p}}^{0}$.

For the "little-oh" case of Theorem 2, we have following corollary.

Corollary 13. Let $0<s \leq n, 1 \leq p<\infty,-1<q+s<\infty$ and let $f \in \mathcal{B}_{\frac{n+1+q}{p}}$. Then the following quantities are equivalent:

(1) $\operatorname{dist}_{\mathcal{B}_{\frac{n+1+q}{p}}}\left(f, \mathcal{B}_{\frac{n+1+q}{p}}^{0}\right)$;

(2) $\operatorname{dist}_{\frac{\mathcal{B}_{\frac{n+1+q}{p}}}{p}}\left(f, F_{0}(p, q, s)\right)$;

(3) $\inf \left\{\varepsilon>0: \frac{\chi_{\tilde{\Omega}_{\varepsilon}(f)}(z)}{\left(1-|z|^{2}\right)^{n+1-s}} \mathrm{~d} v(z)\right.$ is a vanishing $\frac{s}{n}$-Carleson measure $\}$. 
Remark 14. Theorem 2 characterizes the closure of $F(p, q, s)$ in the $\mathcal{B}_{\frac{n+1+q}{p}}$ norm. That is, for $f \in \mathcal{B}_{\frac{n+1+q}{p}}, f$ is in the closure of $F(p, q, s)$ in the $\mathcal{B}_{\frac{n+1+q}{p}}$ norm if and only if for every $\varepsilon>0$,

$$
\int_{\widetilde{\Omega}_{\varepsilon}(f) \cap B(\zeta, r)}\left(1-|z|^{2}\right)^{s-n-1} \mathrm{~d} v(z) \lesssim r^{s}
$$

for all $\zeta \in \mathbf{S}_{n}$ and $r>0$.

The invariant Green's function $G(z, a)$ of $\mathbf{B}_{n}$ is defined by $G(z, a)=g\left(\varphi_{a}(z)\right)$, where

$$
g(z)=\frac{n+1}{2 n} \int_{|z|}^{1}\left(1-t^{2}\right)^{n-1} t^{-2 n+1} \mathrm{~d} t .
$$

The holomorphic function spaces $Q_{s}$ associated with the Green's function is introduced in [4]. For $s>0, Q_{s}$ is defined by

$$
Q_{s}=\left\{f \in H\left(\mathbf{B}_{n}\right): \sup _{a \in \mathbf{B}_{n}} \int_{\mathbf{B}_{n}}|\widetilde{\nabla} f(z)|^{2} G(z, a)^{s} \mathrm{~d} \tau(z)<\infty\right\},
$$

and its subspace $Q_{s, 0}$ is defined by

$$
Q_{s, 0}=\left\{f \in H\left(\mathbf{B}_{n}\right): \lim _{|a| \rightarrow 1} \int_{\mathbf{B}_{n}}|\widetilde{\nabla} f(z)|^{2} G(z, a)^{s} \mathrm{~d} \tau(z)=0\right\},
$$

where $\widetilde{\nabla} f(z)=\nabla\left(f \circ \varphi_{z}\right)(0)$ is the Möbius invariant gradient of $f$, and $\mathrm{d} \tau(z)=(1-$ $\left.|z|^{2}\right)^{-n-1} \mathrm{~d} v(z)$ is the Möbius invariant measure on $\mathbf{B}_{n}$. It is well known that for $n>1$ and $\frac{n-1}{n}<s \leq 1, f \in Q_{s}$ if and only if $|R f(z)|^{2}\left(1-|z|^{2}\right)^{n s+2} \mathrm{~d} \tau(z)$ is an $s$-Carleson measure; $f \in Q_{s, 0}$ if and only if $|R f(z)|^{2}\left(1-|z|^{2}\right)^{n s+2} \mathrm{~d} \tau(z)$ is a vanishing $s$-Carleson measure. Thus $Q_{s}=F(2,1-n, n s)$ and $Q_{s, 0}=F_{0}(2,1-n, n s)$. In particular, when $s=1, Q_{s}=B M O A=F(2,1-n, n)$ and $Q_{s, 0}=V M O A=F_{0}(2,1-n, n)$. Thus, Theorem 2 covers Jone's formula in [1], a part of Zhao's result in [13] and Xu's result in [9].

Acknowledgements. The authors would like to thank the referee for her/his helpful comments and suggestions for improving this manuscript.

\section{References}

[1] Ghatage, P. G., and D. Zheng: Analytic functions of bounded mean oscillation and the Bloch space. - Integral Equations Operators Theory 17, 1993, 501-515.

[2] Lou, Z., and W. Chen: Distances from Bloch functions to $Q_{K}$-type spaces. - Integral Equations Operator Theory 67, 2010, 171-181.

[3] Ortega, J. M., and J. FÀbrega: Pointwise multipliers and corona type decomposition in BMOA. - Ann. Inst. Fourier (Grenoble) 46, 1996, 111-137.

[4] Ouyang, C., W. Yang, and R. ZhaO: Möbius invariant Qp spaces associated with the Green's function on the unit ball of $\mathbf{C}^{n}$. - Pacific J. Math. 182, 1998, 69-99.

[5] QIU, L. and Z. Wu: $s$-Carleson measures and function spaces. - Univ. of Joensuu, Department of Physics and Mathematics, Report Series 12, 2007, 33-55.

[6] TJani, M.: Distance of Bloch functions to the little Bloch space. - Bull. Aust. Math. Soc. 74, 2006, 101-119.

[7] XiAO, J.: Geometric $Q_{p}$ functions. - Birkhauser-Verlag, 2006. 
[8] XIAO, J., and C. YuAn: Analytic Campanato spaces and their compositions. - Indiana Univ. Math. J. (to appear).

[9] Xu, W.: Distances from Bloch functions to some Möbius invariant function spaces in the unit ball of $\mathbf{C}^{n}$. - J. Funct. Spaces Appl. 7, 2009, 91-104.

[10] Yuan, C., and C. Tong: Distance from Bloch-type functions to the analytic space $F(p, q, s)$. - Abstr. Appl. Anal. 2014, ID 610237, 1-7.

[11] Zhang, X., C. He, and F. CaO: The equivalent norms of $F(p, q, s)$ space in $\mathbf{C}^{n}$. - J. Math. Anal. Appl. 401, 2013, 601-610.

[12] ZhaO, R. H.: On a general family of function spaces. - Ann. Acad. Sci. Fenn. Math. Diss. 105, 1996, 1-56.

[13] Zhно, R. H.: Distances from Bloch functions to some Möbius invariant spaces. - Ann. Acad. Sci. Fenn. Math. 33, 2008, 303-313.

[14] ZhaO, R., and K. Zhu: Theory of Bergman spaces in the unit ball of $\mathbf{C}^{n}$. - Mém. Soc. Math. Fr. (N.S.) 115, 2008.

[15] ZHU, K.: Spaces of holomorphic functions in the unit ball. - Grad. Texts in Math. 226, Springer, New York, 2005.

[16] ZHU, K.: Operator theory in function spaces. - Amer. Math. Soc., 2007.

Received 13 May $2014 \bullet$ Accepted 19 September 2014 